\title{
THE PROOF-THEORETIC ANALYSIS OF TRANSFINITELY ITERATED FIXED POINT THEORIES
}

\author{
GERHARD JÄGER, REINHARD KAHLE, ANTON SETZER, AND THOMAS STRAHM
}

\begin{abstract}
This article provides the proof-theoretic analysis of the transfinitely iterated fixed point theories $\widehat{I D}_{\alpha}$ and $\widehat{I D}_{<\alpha}$ : the exact proof-theoretic ordinals of these systems are presented.
\end{abstract}

\$1. Introduction. The transfinitely iterated fixed point theories $\widehat{\mathrm{ID}}_{\alpha}$ are relatives of the better known theories ID $\alpha$ for iterated inductive definitions. These latter theories have been extensively studied during the last years (cf., e.g., Buchholz et al. [1]) and their proof-theoretic analysis has been carried through in all detail.

The basic axioms of ID $\alpha$ provide hierarchies of least (definable) fixed points of $\alpha$ times iterated positive inductive definitions given by arithmetic operator forms. In the case of the fixed point theories $\widehat{I D}_{\alpha}$, on the other hand, one confines oneself to hierarchies of arbitrary fixed points of the corresponding inductive definitions and drops the requirement for minimality.

The finitely iterated fixed point theories $\widehat{I D}_{n}$ were first introduced in Feferman [5] in connection with his proof of Hancock's conjecture. Among other things, it is shown in this article that the proof-theoretic ordinal of $\widehat{\mathrm{ID}}_{n}$ is $\alpha_{n}$ for $\alpha_{0}:=\varepsilon_{0}$ and $\alpha_{n+1}:=\varphi \alpha_{n} 0$. Hence, the union of all $\widehat{\mathrm{ID}}_{n}$ for $n<\omega$, i.e., the system $\widehat{\mathrm{ID}}_{<\omega}$, has proof-theoretic ordinal $\Gamma_{0}$, thus providing a framework for predicative mathematics.

In this article we make the step to transfinite iterations of fixed points and are interested in the theories $\widehat{\mathrm{ID}}_{\alpha}$ for $\alpha \geq \omega$. It is a technical paper, which establishes the proof-theoretic ordinals of these systems. The relationship between transfinitely iterated fixed point theories and subsystems of second order arithmetic and the role of transfinitely iterated fixed point theories for metapredicativity in general are only briefly addressed in the conclusion.

The plan of this paper is as follows. In Section 2 we discuss some ordinal-theoretic preliminaries; namely, we sketch an ordinal notation system which is based on $n$ ary $\varphi$ functions. In Section 3 we introduce the theories $\widehat{I D}_{\alpha}$ and we state the Main Theorem about the proof-theoretic ordinal of $\widehat{I D}_{\alpha}$. Section 4 is devoted to the definition of transfinitely iterated theories for self-reflecting truth $\mathrm{SRT}_{\alpha}$. These systems are contained in $\widehat{\mathrm{ID}}_{\alpha}$, and greatly facilitate the wellordering proofs for the latter, which we will present in Section 5. A full cut elimination procedure for $\widehat{\mathrm{ID}}_{\alpha}$ is provided in Section 6. Finally, we discuss some related topics in Section 7.

Received February 10, 1997.

(C) 1999. Association for Symbolic Logic $0022-4812 / 99 / 6401-0005 / \$ 2.50$ 
This paper grew out of intensive discussions among its authors and the results have been conjectured or proved by all of them. The presentation of the material, however, focusing on two main lemmas, goes to a large extent back to Thomas Strahm.

§2. Ordinal-theoretic preliminaries. It is the aim of this section to discuss a few ordinal-theoretic facts which will be relevant in the sequel. Namley, we sketch an ordinal notation system which is based on $n$-ary $\varphi$ functions.

The standard notation system up to the Feferman-Schütte ordinal $\Gamma_{0}$ makes use of the usual Veblen hierarchy generated by the binary function $\varphi$, starting off with the function $\varphi 0 \beta=\omega^{\beta}$, cf. Pohlers [12] and Schütte [15]. However, ordinals up to $\Gamma_{0}$ are no longer sufficient for an ordinal analysis of transfinitely iterated fixed point theories.

Here the natural notations are obtained by means of a ternary $\varphi$ function. One simply generalizes the definition principle of the usual binary $\varphi$ function and generates the ternary $\varphi$ function inductively as follows:

(i) $\varphi 0 \beta \gamma$ is just $\varphi \beta \gamma$.

(ii) If $\alpha>0$, then $\varphi \alpha 0 \gamma$ denotes the $\gamma$ th ordinal which is strongly critical with respect to all functions $\lambda \xi, \eta \cdot \varphi \alpha^{\prime} \xi \eta$ for $\alpha^{\prime}<\alpha$.

(iii) If $\alpha>0$ and $\beta>0$, then $\varphi \alpha \beta \gamma$ denotes the $\gamma$ th common fixed point of the functions $\lambda \xi . \varphi \alpha \beta^{\prime} \xi$ for $\beta^{\prime}<\beta$.

For example, $\varphi 10 \alpha$ is $\Gamma_{\alpha}$, and more generally, $\varphi 1 \alpha \beta$ denotes a Veblen hierarchy over $\lambda \alpha . \Gamma_{\alpha}$. It is straightforward how to extend these ideas in order to obtain $\varphi$ functions of all finite arities greater than 3 , and even further to Schütte's Klammersymbole [14].

Let $\Phi_{0}$ be the least ordinal greater than 0 which is closed under all $n$-ary $\varphi$ functions with $n \geq 2$. In the following we confine ourselves to the standard notation system which is based on these $n$-ary $\varphi$ functions $(n \geq 2)$. Since the exact definition of such a system is a straightforward generalization of the notation system for $\Gamma_{0}$ (cf. $[12,15]$ ), we do not go into details here. We write $\prec$ for the corresponding primitive recursive wellordering and assume without loss of generality that the field of $\prec$ is the set of all natural numbers and 0 is the least element with respect to $\prec$. Hence, each natural number codes an ordinal less than $\Phi_{0}$. Moreover, there exist primitive recursive functions acting on these codes which correspond to ordinal operations such as plus, times, exponentiation and $\varphi$.

When working with formal theories, it is often convenient in order to simplify notation to use ordinals and ordinal operations instead of their codes and primitive recursive analogues. Then (for example) $\omega$ and $\omega+\omega$ stand for the natural numbers whose order type with respect to $\prec$ are $\omega$ and $\omega+\omega$.

§3. The theories $\widehat{I D}_{\alpha}$. It is the aim of this section to give a precise definition of transfinitely iterated fixed point theories $\widehat{\mathrm{ID}}_{\alpha}$ for $\alpha<\Phi_{0}$. In the following we let $\mathscr{L}$ denote the language of first order arithmetic. $\mathscr{L}$ includes number variables $(a, b$, $c, d, e, f, g, h, u, v, w, x, y, z, \ldots)$, symbols for all primitive recursive functions 
and relations, as well as a unary relation symbol $U$ whose status will become clear below. Furthermore, there is a symbol $\sim$ for forming negative literals. ${ }^{1}$

The number terms $(r, s, t, \ldots)$ of $\mathscr{L}$ are defined as usual. Positive literals of $\mathscr{L}$ are all expressions $R\left(s_{1}, \ldots, s_{n}\right)$ for $R$ a symbol for an $n$-ary primitive recursive relation as well as expressions of the form $U(s)$. The negative literals of $\mathscr{L}$ have the form $\sim E$ so that $E$ a positive literal. The formulas $(A, B, C, \ldots)$ of $\mathscr{L}$ are now generated from the positive and negative literals of $\mathscr{L}$ by closing against disjunction, conjunction, as well as existential and universal number quantification. The negation $\neg A$ of an $\mathscr{L}$ formula $A$ is defined by making use of De Morgan's laws and the law of double negation. Moreover, the remaining logical connectives are abbreviated as usual.

If $P$ and $Q$ are fresh unary relation symbols, then we let $\mathscr{L}(P, Q)$ denote the extension of $\mathscr{L}$ by $P$ and $Q$. We call an $\mathscr{L}(P, Q)$ formula $P$-positive, if it has no subformulas of the form $\sim P(t)$. A $P$-positive $\mathscr{L}(P, Q)$ formula which contains at most $x$ and $y$ free is called an inductive operator form, and we let $\mathscr{A}(P, Q, x, y)$ range over such forms.

We set for all primitive recursive relations $\sqsubset$, all formulas $A(x)$ and terms $s$ :

$$
\begin{aligned}
\operatorname{Prog}(\sqsubset, A) & :=(\forall x)[(\forall y)(y \sqsubset x \rightarrow A(y)) \rightarrow A(x)], \\
\operatorname{TI}(\sqsubset, A) & :=\operatorname{Prog}(\sqsubset, A) \rightarrow(\forall x) A(x), \\
\operatorname{TI}(\sqsubset, s, A) & :=\operatorname{Prog}(\sqsubset, A) \rightarrow(\forall x \sqsubset s) A(x) .
\end{aligned}
$$

In the sequel we write $\operatorname{Prog}(A)$ and $\operatorname{TI}(s, A)$ instead of $\operatorname{Prog}(\prec, A)$ and $\operatorname{TI}(\prec, s, A)$, respectively. If we want to stress the relevant induction variable of the formula $A$, we sometimes write $\operatorname{Prog}(\lambda x \cdot A(x))$ instead of $\operatorname{Prog}(A)$.

In order to formulate the theories $\widehat{\mathbb{D}}_{\alpha}$ for $\alpha<\Phi_{0}$, we add to the first order language $\mathscr{L}$ a new unary relation symbol $P^{\mathscr{A}}$ for each inductive operator form $\mathscr{A}(P, Q, x, y)$ and denote this new language by $\mathscr{L}_{\text {fix. }}$. We write $P_{s}^{\mathscr{A}}(t)$ for $P^{\mathscr{A}}(\langle t, s\rangle)$ and $P_{\prec s}^{\mathscr{A}}(t)$ for $t=\left\langle(t)_{0},(t)_{1}\right\rangle \wedge(t)_{1} \prec s \wedge P^{\mathscr{A}}(t)$; here $\langle\cdot, \cdot\rangle$ denotes a primitive recursive coding function with associated projections $(\cdot)_{0}$ and $(\cdot)_{1}$.

For each ordinal $\alpha$ less than $\Phi_{0}$ we now introduce the theory $\widehat{I D}_{\alpha}$ for $\alpha$ times iterated fixed points. The axioms of $\widehat{\mathrm{ID}}_{\alpha}$ comprise (i) the axioms of Peano arithmetic with the scheme of complete induction for all formulas of $\mathscr{L}_{\text {fix }}$, (ii) the fixed point axioms

$$
(\forall a \prec \alpha)(\forall x)\left[P_{a}^{\mathscr{A}}(x) \leftrightarrow \mathscr{A}\left(P_{a}^{\mathscr{A}}, P_{\prec a}^{\mathscr{A}}, x, a\right)\right]
$$

for all inductive operator forms $\mathscr{A}(X, Y, x, y)$, as well as (iii) the axioms $\operatorname{TI}(\alpha, A)$ for all $\mathscr{L}_{\text {fix }}$ formulas $A$.

As usual we call an ordinal $\alpha$ provable in a theory $T$, if there is a primitive recursive wellordering $\sqsubset$ of order type $\alpha$ so that $\mathrm{T} \vdash \operatorname{TI}(\sqsubset, U)$. The least ordinal which is not provable in $\mathrm{T}$ is called the proof-theoretic ordinal of $\mathrm{T}$ and is denoted by $|\mathrm{T}|$.

We are ready to formulate the main theorem of this article. For that purpose we let $\varepsilon(\alpha)$ denote the least $\varepsilon$ number greater than $\alpha$. Moreover, the ordinals $(\alpha \mid m)$ are inductively defined by

$$
(\alpha \mid 0):=\varepsilon(\alpha), \quad(\alpha \mid m+1):=\varphi(\alpha \mid m) 0 .
$$

${ }^{1}$ This formulation of the language is chosen for the Tait-style reformulation of our systems in Section 6. 
MAIN TheOREM. Assume that $\alpha$ is an ordinal less than $\Phi_{0}$ given in the form

$$
\alpha=\omega^{1+\alpha_{n}}+\omega^{1+\alpha_{n-1}}+\cdots+\omega^{1+\alpha_{1}}+m
$$

for ordinals $\alpha_{n} \geq \alpha_{n-1} \geq \cdots \geq \alpha_{1}$ and $m<\omega$. Then we have

$$
\left|\widehat{\mid D}_{\alpha}\right|=\varphi 1 \alpha_{n}\left(\varphi 1 \alpha_{n-1}\left(\cdots \varphi 1 \alpha_{1}(\alpha \mid m)\right) \cdots\right) \text {. }
$$

Of course, the restriction to ordinals $\alpha$ less than $\Phi_{0}$ is not essential here; it just stems from the notation system which we have chosen for the purpose of this article. In the sequel we suppose that all our ordinals are less than $\Phi_{0}$.

A first step towards the proof of the main theorem consists in introducing transfinitely iterated theories for self-reflecting truth, which will greatly facilitate the wellordering proofs for $\widehat{\mathrm{ID}}_{\alpha}$ in Section 5 .

\$4. Transfinitely iterated theories for self-reflecting truth. The notion of selfreflecting truth is a well-developed idea in proof theory. Theories for formalized and self-reflecting truth are presented and studied for example in Cantini $[3,4]$ and Feferman [6].

In this section we introduce transfinitely iterated theories for self-reflecting truth $\mathrm{SRT}_{\alpha}$ for $\alpha<\Phi_{0}$. It will be immediate that $\mathrm{SRT}_{\alpha}$ can be modeled in $\widehat{\mathrm{ID}}_{\alpha}$. In the next section we will see that theories for self-reflecting truth provide a natural framework for carrying through wellordering proofs.

The theories $\mathrm{SRT}_{\alpha}$ are formulated in the language $\mathscr{L}_{\text {srt }}$, which extends $\mathscr{L}$ by two binary relation symbols $T$ (for "true") and $F$ (for "false"). The terms of $\mathscr{L}_{\text {srt }}$ are just the $\mathscr{L}$ terms; the formulas of $\mathscr{L}_{\text {stt }}$ are given in a straightforward manner, taking into account the new atoms $T(s, t)$ (" $t$ is true on level $s$ ") and $F(s, t)$ (" $t$ is false on level $s ")$ as well as their complementations. In the following we often write $T_{s}(t)$ and $F_{s}(t)$ instead of $T(s, t)$ and $F(s, t)$, respectively.

If $\alpha$ is an ordinal less than $\Phi_{0}$, then we obtain the sublanguage $\mathscr{L}_{\mathrm{srt}}^{\alpha}$ of $\mathscr{L}_{\text {st }}$ by restricting atoms of the form $T_{s}(t)$ and $F_{s}(t)$ to closed terms $s$ with value less than or equal to $\alpha$ (in the sense of our notation system). Hence, variables are not allowed in the first place of the relation symbols $T$ and $F$ in $\mathscr{L}_{\text {srt }}^{\alpha}$ formulas.

In order to describe transfinitely iterated truth theories below, we presuppose a standard Gödelization of the languages $\mathscr{L}_{\text {srt }}^{\alpha}$, uniformly in $\alpha<\Phi_{0}$. In particular, we have Gödel numbers $\ulcorner t\urcorner$ and $\ulcorner A\urcorner$ for each $\mathscr{L}$ term $t$ and each $\mathscr{L}_{\text {srt }}^{\alpha}$ formula $A$. Moreover, we will use the following $\mathscr{L}$ definable functions and predicates on Gödel numbers: CTer $(x)$ (" $x$ is closed term of $\mathscr{L} ")$; $\operatorname{For}_{n}(f, a)$ (" $f$ is an $\mathscr{L}_{\text {srt }}^{u}$ formula with at most $n$ free variables"); $\operatorname{Atm}(f, a)$ (" $f$ is a positive literal of $\mathscr{L}_{\mathrm{srt}}^{a}$ "); $\operatorname{num}(x)$ ("the $x$ th numeral of $\mathscr{L}$ "); $\operatorname{val}(z)$ ("the value of the closed term $z$ "); neg $(f)$ ("the negation of the positive atom $f$ "); and $(f, g)$ ("the conjunction of $f$ and $g$ "); $\operatorname{or}(f, g)$ is defined analogously; all $(x, f)$ ("the universal quantification of $f$ with respect to the $x$ th variable of $\left.\mathscr{L}^{\prime \prime}\right)$; $\operatorname{ext}(x, f)$ for existential quantification is defined analogously. In the following we write $\operatorname{Sen}(f, a)$ instead of $\operatorname{For}_{0}(f, a)$ and $\dot{x}$ instead of num $(x)$. Finally, if $R$ is an $\mathscr{L}$ relation symbol, CTer $(x)$ and CTer $(a)$, then expressions like $\ulcorner R(x)\urcorner,\left\ulcorner T_{a}(x)\right\urcorner$, and $\left\ulcorner F_{a}(x)\right\urcorner$ have their obvious meaning.

We conclude our tedious but standard preliminary remarks by adopting some conventions concerning substitution. If $\operatorname{For}_{n}(f, a)$ and $\operatorname{CTer}\left(x_{1}\right), \ldots, \operatorname{CTer}\left(x_{n}\right)$, then $f\left(x_{1}, \ldots, x_{n}\right)$ denotes (the code of) the formula which is obtained from $f$ by 
substituting the $i$ th free variable of $f$ by $x_{i}$; in particular, $\operatorname{Sen}\left(f\left(x_{1}, \ldots, x_{n}\right), a\right)$. Similarly, if the free variables of $A$ are among $x_{1}, \ldots, x_{n}$, then $\left\ulcorner A\left(\dot{x}_{1}, \ldots, \dot{x}_{n}\right)\right\urcorner$ is an abbreviation for $\ulcorner A\urcorner\left(\dot{x}_{1}, \ldots, \dot{x_{n}}\right)$.

If $\alpha$ is an ordinal less than $\Phi_{0}$, then the theory SRT ${ }_{\alpha}$ for $\alpha$ times iterated selfreflecting truth comprises (i) the axioms of Peano arithmetic with the scheme of complete induction for all formulas of $\mathscr{L}_{\text {stt }}$, (ii) the axioms $\operatorname{TI}(\alpha, A)$ for all $\mathscr{L}_{\text {st }}$ formulas $A$, and (iii) the following axiom groups I to III.

I. Atomic truth.

(1) For each relation symbol $R$ of $\mathscr{L}$ :

$$
\begin{aligned}
\operatorname{CTer}\left(x_{1}\right) \wedge & \cdots \wedge \operatorname{CTer}\left(x_{n}\right) \wedge a \prec \alpha \\
\rightarrow\left[T_{a}\left(\left\ulcorner R\left(x_{1}, \ldots, x_{n}\right)\right\urcorner\right) \leftrightarrow R\left(\operatorname{val}\left(x_{1}\right), \ldots, \operatorname{val}\left(x_{n}\right)\right)\right] & \\
& \wedge\left[F_{a}\left(\left\ulcorner R\left(x_{1}, \ldots, x_{n}\right)\right\urcorner\right) \leftrightarrow \neg R\left(\operatorname{val}\left(x_{1}\right), \ldots, \operatorname{val}\left(x_{n}\right)\right)\right] .
\end{aligned}
$$

(2) $\quad C \operatorname{Ter}(x) \wedge C \operatorname{Ter}(b) \wedge \operatorname{val}(b) \prec a \prec \alpha$

$$
\begin{aligned}
\rightarrow\left[T_{a}\left(\left\ulcorner T_{b}(x)\right\urcorner\right)\right. & \left.\leftrightarrow T_{\operatorname{val}(b)}(\operatorname{val}(x))\right] \wedge\left[T_{a}\left(\left\ulcorner F_{b}(x)\right\urcorner\right) \leftrightarrow F_{\operatorname{val}(b)}(\operatorname{val}(x))\right] \\
\wedge\left[F_{a}\left(\left\ulcorner T_{b}(x)\right\urcorner\right)\right. & \left.\leftrightarrow \neg T_{\operatorname{val}(b)}(\operatorname{val}(x))\right] \wedge\left[F_{a}\left(\left\ulcorner F_{b}(x)\right\urcorner\right) \leftrightarrow \neg F_{\operatorname{val}(b)}(\operatorname{val}(x))\right] .
\end{aligned}
$$

II. Composed truth.

(3) $\operatorname{Atm}(f, a) \wedge a \prec \alpha \rightarrow\left[T_{a}(\operatorname{neg}(f)) \leftrightarrow F_{a}(f)\right] \wedge\left[F_{a}(\operatorname{neg}(f)) \leftrightarrow T_{a}(f)\right]$,

(4) $\operatorname{Sen}(f, a) \wedge \operatorname{Sen}(g, a) \wedge a \prec \alpha$

$\rightarrow\left[T_{a}(\operatorname{and}(f, g)) \leftrightarrow T_{a}(f) \wedge T_{a}(g)\right] \wedge\left[F_{a}(\operatorname{and}(f, g)) \leftrightarrow F_{a}(f) \vee F_{a}(g)\right]$,

(5) dual clauses for disjunction

(6) $\operatorname{Sen}(\operatorname{all}(v, f), a) \wedge a \prec \alpha$

$$
\rightarrow\left[T_{a}(\operatorname{all}(v, f)) \leftrightarrow(\forall x) T_{a}(f(\dot{x}))\right] \wedge\left[F_{a}(\operatorname{all}(v, f)) \leftrightarrow(\exists x) F_{a}(f(\dot{x}))\right],
$$

(7) dual clauses for existential quantification.

\section{Self reflecting truth.}

(8) $\operatorname{CTer}(x) \wedge C \operatorname{Ter}(a) \wedge \operatorname{val}(a) \prec \alpha$

$$
\begin{aligned}
& \rightarrow\left[T_{\operatorname{val}(a)}\left(\left\ulcorner T_{a}(x)\right\urcorner\right) \leftrightarrow T_{\operatorname{val}(a)}(\operatorname{val}(x))\right] \wedge\left[T_{\operatorname{val}(a)}\left(\left\ulcorner F_{a}(x)\right\urcorner\right) \leftrightarrow F_{\operatorname{val}(a)}(\operatorname{val}(x))\right] \\
& \wedge\left[F_{\operatorname{val}(a)}\left(\left\ulcorner T_{a}(x)\right\urcorner\right) \leftrightarrow F_{\operatorname{val}(a)}(\operatorname{val}(x))\right] \wedge\left[F_{\operatorname{val}(a)}\left(\left\ulcorner F_{a}(x)\right\urcorner\right) \leftrightarrow T_{\operatorname{val}(a)}(\operatorname{val}(x))\right] .
\end{aligned}
$$

It is completely straightforward to model the theory $\mathrm{SRT}_{\alpha}$ for $\alpha$ times iterated self-reflecting truth by means of a fixed point hierarchy of length $\alpha$; for a similar argument in the case of one times iterated truth theories, the curious reader is advised to consult Feferman [6]. Therefore, we can state the following proposition without proof.

Proposition 1. There exists an embedding of $\mathrm{SRT}_{\alpha}$ into $\widehat{\mathrm{ID}}_{\alpha}$.

Let us now turn to some crucial consequences of theories for self-reflecting truth. They will implicitly be used in the wellordering proofs of the next section. We first 
observe that $T_{a}$ and $F_{a}$ are complementary on sentences of level less than $a$. This is easily established by (formal) induction on the build up of such sentences.

Proposition 2. The following is a theorem of $\mathrm{SRT}_{\alpha}$ :

$$
(\forall a, b, f)\left[\operatorname{Sen}(f, b) \wedge b \prec a \prec \alpha \rightarrow\left(T_{a}(f) \leftrightarrow \neg F_{a}(f)\right)\right] .
$$

If $a$ is a variable of $\mathscr{L}$, then we call an $\mathscr{L}_{\text {srt }}$ formula $\left(T_{a}, F_{a}\right)$ positive, if it is built from $\mathscr{L}$ literals which do not contain $a$, atoms of the form $T_{a}(t), F_{a}(t)$ so that $a$ does not occur in $t$, and by closing against conjunction, disjunction as well as quantification with respect to variables different from $a$. Hence, in a $\left(T_{a}, F_{a}\right)$ positive formula only positive occurrences of $T_{a}$ and $F_{a}$ are allowed, and in addition, the variable $a$ is used as an (unquantified) level variable only. If $A\left(a, x_{1}, \ldots, x_{n}\right)$ is such a $\left(T_{a}, F_{a}\right)$-positive formula with level variable $a$ and additional free variables among $x_{1}, \ldots, x_{n}$, then there is associated uniformly in a valuation $\dot{a}, \dot{x}_{1}, \ldots, \dot{x_{n}}$ for $a, x_{1}, \ldots, x_{n}$ a Gödel number $\left\ulcorner A\left(\dot{a}, \dot{x_{1}}, \ldots, \dot{x}_{n}\right)\right\urcorner$ of the corresponding $\mathscr{L}_{\text {srt }}^{a}$ formula. The following proposition is characteristic for selfreflective truth theories (cf., e.g., Cantini [3]) and is easily established by induction on the complexity of $A$.

Proposition 3. Assume that $A\left(a, x_{1}, \ldots, x_{n}\right)$ is a $\left(T_{a}, F_{a}\right)$ positive formula with at most $a, x_{1}, \ldots, x_{n}$ free. Then $\mathrm{SRT}_{\alpha}$ proves

$$
(\forall a \prec \alpha)\left(\forall x_{1}, \ldots, x_{n}\right)\left[T_{a}\left(\left\ulcorner A\left(\dot{a}, \dot{x}_{1}, \ldots, \dot{x}_{n}\right)\right\urcorner\right) \leftrightarrow A\left(a, x_{1}, \ldots, x_{n}\right)\right] .
$$

Finally, there is a natural notion of (ramified) set in our truth theoretic framework, namely, sets of natural numbers are understood as propositional functions. More formally, we define the notions " $f$ is a set of level $a$ ", $f \in S_{a}$, and " $x$ is an element of a set $f$ of level $a$ ", $x \in_{a} f$, as follows.

$$
\begin{aligned}
& f \in S_{a}:=\operatorname{For}_{1}(f, a) \wedge(\forall x)\left(T_{a}(f(\dot{x})) \leftrightarrow \neg F_{a}(f(\dot{x})),\right. \\
& x \in \in_{a} f:=T_{a}(f(\dot{x})) .
\end{aligned}
$$

In the sequel we often write $x \in f$ instead of $x \in_{a} f$ if it is clear from the context that $f$ is a set of level $a$. Moreover, if $A(P)$ is an $\mathscr{L}(P)$ formula, then we write $A(f)$ for the $\mathscr{L}_{\text {srt }}$ formula which is obtained from $A$ by replacing each atom of the form $P(t)$ by $t \in f$.

In the next section we turn to the wellordering proofs for $\widehat{\mathrm{ID}}_{\alpha}$, which we will carry through in $\mathrm{SRT}_{\alpha}$.

§5. Wellordering proofs for $\widehat{I D}_{\alpha}$. This section is devoted to the wellordering proofs for the theories $\widehat{\mathrm{ID}}_{\alpha}$ for each $\alpha<\Phi_{0}$. As we have announced in the last section, we carry through these proofs in $\mathrm{SRT}_{\alpha}$, which is contained in $\widehat{\mathrm{ID}}_{\alpha}$ by Proposition 1 . In the sequel we presuppose that the reader is familiar with wellordering proofs for finitely iterated fixed point theories, i.e., wellordering proofs below $\Gamma_{0}$, and we assume that she or he has some experience in working with partial truth theories. Useful references concerning these matters are, for example, Cantini [3], Feferman [6], and Schütte [15].

In the following we adopt some conventions concerning limit notations in our notation system, which will be needed in the proof of Main Lemma I below. We let Lim denote the primitive recursive set of limit notations and presuppose a primitive 
recursively given canonical fundamental sequence $(\ell[n]: n \in \mathbb{N})$ for each limit notation $\ell$. For technical reasons we assume that $\ell[0]>0$. Since the definition of fundamental sequences is straightforward in the setting of $\varphi$ functions (cf., e.g., [6]), we do not give details here and refer to the proof of Main Lemma I.

Crucial for carrying out wellordering proofs in transfinitely iterated theories for self-reflecting truth is the very natural notion $I^{c}(a)$ of transfinite induction up to a for all sets of level less than $c$, which is given as follows:

$$
I^{c}(a):=(\forall b \prec c)\left(\forall f \in S_{b}\right) \operatorname{TI}(f, a) .
$$

The next lemma states that $I^{\ell}(a)$ can be represented by a set of level $\ell$ for limit notations $\ell$. Its proof is straightforward and, therefore, we omit it.

LEMMA 4. The following is a theorem of $\mathrm{SRT}_{\alpha}$ :

$$
(\forall \ell)\left[\operatorname{Lim}(\ell) \wedge \ell \prec \alpha \rightarrow\left(\exists f \in S_{\ell}\right)(\forall a)\left(I^{\ell}(a) \leftrightarrow a \in_{\ell} f\right)\right] .
$$

The following lemma is crucial for the base case in Main Lemma I below. We do not give its proof here, since the relevant arguments can easily be extracted from Schütte [15, pp. $184 \mathrm{ff}$.] and using standard techniques for working with partial truth theories as they are presented in Cantini [3] and Feferman [6].

LEMMA 5. The following is a theorem of SRT $\mathrm{S}_{\alpha}$

$$
(\forall \ell, a)\left[\operatorname{Lim}(\ell) \wedge \ell \preceq \alpha \wedge I^{\ell}(a) \rightarrow I^{\ell}(\varphi a 0)\right] .
$$

As an immediate consequence of this lemma we obtain the following corollary.

COROLLARY 6. The following is a theorem of $\mathrm{SRT}_{\alpha}$ :

$$
(\forall \ell)\left[\operatorname{Lim}(\ell) \wedge \ell \preceq \alpha \rightarrow \operatorname{Prog}\left(\lambda a . I^{\ell}\left(\Gamma_{a}\right)\right)\right] .
$$

The formulation of Main Lemma I makes crucial use of the binary relation $\uparrow$, which is given as follows:

$$
a \uparrow b:=(\exists c, \ell)(\operatorname{Lim}(\ell) \wedge b=c+a \cdot \ell) .
$$

We are now ready to state the main lemma of this section. It has some similarities with Lemma 5.3.1 of Feferman [6] in a wellordering proof below $\Gamma_{0}$.

LeMma 7 (Main Lemma I). Let the formula $\operatorname{Main}_{\alpha}(a)$ be defined as follows:

$$
\operatorname{Main}_{\alpha}(a):=(\forall b, c)\left[c \preceq \alpha \wedge \omega^{1+a} \uparrow c \wedge I^{c}(b) \rightarrow I^{c}(\varphi 1 a b)\right] .
$$

Then SRT $T_{\alpha}$ proves $\operatorname{Prog}\left(\lambda a . \operatorname{Main}_{\alpha}(a)\right)$.

Proof. For the proof of this lemma we work informally in $\mathrm{SRT}_{\alpha}$. We break the proof of $\operatorname{Prog}\left(\lambda a\right.$. $\left.\operatorname{Main}_{\alpha}(a)\right)$ into three cases by showing:

(a) $\operatorname{Main}_{\alpha}(0)$

(b) $\operatorname{Main}_{\alpha}(a) \rightarrow \operatorname{Main}_{\alpha}(a+1)$;

(c) $\operatorname{Lim}(a) \wedge(\forall w) \operatorname{Main}_{\alpha}(a[w]) \rightarrow \operatorname{Main}_{\alpha}(a)$.

It is clear that $\operatorname{Prog}\left(\lambda a . \operatorname{Main}_{\alpha}(a)\right)$ follows from (a)-(c).

(a) $\operatorname{Main}_{\alpha}(0)$. Let us assume that $c=c_{0}+\omega \cdot \ell$ for a limit notation $\ell$. We have to show

$$
I^{c_{0}+\omega \cdot \ell}(b) \rightarrow I^{c_{0}+\omega \cdot \ell}\left(\Gamma_{b}\right)
$$


Towards this end, assume $I^{c_{0}+\omega \cdot \ell}(b)$. It is sufficient to verify $I^{c_{0}+\omega \cdot \ell[u]}\left(\Gamma_{b}\right)$ for each natural number $u$. Since $\ell[u]>0$ we have that $c_{0}+\omega \cdot \ell[u]$ is always limit, and hence we obtain by the previous corollary that

$$
\operatorname{Prog}\left(\lambda a . I^{c_{0}+\omega \cdot \ell[u]}\left(\Gamma_{a}\right)\right)
$$

for each $u$. But we have that $I^{c_{0}+\omega \cdot \ell[u]}(a)$ can be represented by a set of level $c_{0}+\omega \cdot \ell[u]$, so that $I^{c_{0}+\omega \cdot \ell}(b)$ and (2) immediately imply

$$
I^{c_{0}+w \cdot \ell[u]}\left(\Gamma_{b}\right)
$$

for each natural number $u$. This finishes part (a) of our proof.

(b) $\operatorname{Main}_{\alpha}(a) \rightarrow \operatorname{Main}_{\alpha}(a+1)$. Let us assume $\operatorname{Main}_{\alpha}(a)$, i.e.

$$
(\forall d, e)\left[d \preceq \alpha \wedge \omega^{1+a} \uparrow d \wedge I^{d}(e) \rightarrow I^{d}(\varphi 1 a e)\right] .
$$

We want to establish $\operatorname{Main}_{\alpha}(a+1)$. So assume

$$
c \preceq \alpha \wedge c=c_{0}+\omega^{1+a+1} \cdot \ell \wedge I^{c}(b) .
$$

We have to show $I^{c}(\varphi 1(a+1) b)$. Again it is sufficient to establish

$$
I^{c_{0}+\omega^{1+a+1} \cdot \ell[u]}(\varphi 1(a+1) b)
$$

for each natural number $u$. We set $c[u]:=c_{0}+\omega^{1+a+1} \cdot \ell[u]$ and readily observe that $\omega^{1+a} \uparrow c[u]$ for each $u$. Hence, we can derive from (4)

$$
(\forall u)(\forall e)\left[I^{c[u]}(e) \rightarrow I^{c[u]}(\varphi 1 a e)\right] .
$$

In the following we want to establish

$$
(\forall u) \operatorname{Prog}\left(\lambda e . I^{c}[u](\varphi 1(a+1) e)\right) .
$$

The proof of $(8)$ requires the verification of

$$
\begin{gathered}
I^{c[u]}(\varphi 1(a+1) 0), \\
I^{c[u]}(\varphi 1(a+1) e) \rightarrow I^{c[u]}(\varphi 1(a+1)(e+1)), \\
\operatorname{Lim}(e) \wedge\left(\forall e^{\prime} \prec e\right) I^{c[u]}\left(\varphi 1(a+1) e^{\prime}\right) \rightarrow I^{c[u]}(\varphi 1(a+1) e) .
\end{gathered}
$$

In order to verify (9), observe that we are given a fundamental sequence $z_{w}=$ $\varphi 1(a+1) 0[w]$ for $\varphi 1(a+1) 0$, where $z_{0}=1$ and $z_{w+1}=\varphi 1 a z_{w}$. Hence, (9) follows from (7) by ordinary induction. As to (10), we have a fundamental sequence $z_{w}=\varphi 1(a+1)(e+1)[u]$ for $\varphi 1(a+1)(e+1)$ with $z_{0}=\varphi 1(a+1) e+1$ and $z_{w+1}=\varphi 1 a z_{w}$. Again the claim follows from (7) by ordinary induction. Finally, for (11), we observe that if $\operatorname{Lim}(e)$, then $\varphi 1(a+1) e$ is the supremum over $e^{\prime} \prec e$ of $\varphi 1(a+1) e^{\prime}$, hence the claim is immediate in this case. Thus we have finished the verification of $(8)$. Since $I^{c[u]}(\varphi 1(a+1) e)$ can be represented by a set of level $c[u]$ for each $u$, and we know $I^{c}(b)$ by $(5)$, we are now in a position to conclude from (8) that

$$
(\forall u) I^{c[u]}(\varphi 1(a+1) b) .
$$

But this is exactly (6) and, hence, part (b) of our proof is finished. 
(c) $\operatorname{Lim}(a) \wedge(\forall w) \operatorname{Main}_{\alpha}(a[w]) \rightarrow \operatorname{Main}_{\alpha}(a)$. Let us assume

$$
\begin{gathered}
\operatorname{Lim}(a) \wedge(\forall w) \operatorname{Main}_{\alpha}(a[w]), \\
c \preceq \alpha \wedge c=c_{0}+\omega^{1+a} \cdot \ell \wedge I^{c}(b) .
\end{gathered}
$$

Observe that we have $\omega^{1+a}=\omega^{a}$ since $a$ is limit. We have to show $I^{c}(\varphi 1 a b)$. Indeed, it is enough to establish

$$
I^{c_{0}+\omega^{a} \cdot \ell[u]}(\varphi 1 a b)
$$

for each natural number $u$. If we set $c[u]:=c_{0}+\omega^{a} \cdot \ell[u]$, then one readily sees that for each $w$, we can write $c[u]$ in the form

$$
c[u]=c_{0}+\omega^{1+a[w]} \cdot \ell_{w}
$$

for a suitable limit notation $\ell_{w}$ depending on $w$ (and $\ell[u]$ ). Hence, we have that $\omega^{1+a[w]} \uparrow c[u]$ for each $w$ and, therefore, we can derive from (13) that

$$
(\forall u, w, e)\left[I^{c[u]}(e) \rightarrow I^{c[u]}(\varphi 1 a[w] e)\right]
$$

In a further step we now want to derive

$$
(\forall u) \operatorname{Prog}\left(\lambda e . I^{c[u]}(\varphi 1 a e)\right) \text {. }
$$

Again this breaks into three subcases (19)-(21), namely

$$
\begin{gathered}
I^{c[u]}(\varphi 1 a 0), \\
I^{c[u]}(\varphi 1 a e) \rightarrow I^{c[u]}(\varphi 1 a(e+1)), \\
\operatorname{Lim}(e) \wedge\left(\forall e^{\prime} \prec e\right) I^{c[u]}\left(\varphi 1 a e^{\prime}\right) \rightarrow I^{c[u]}(\varphi 1 a e) .
\end{gathered}
$$

As to (19), we have a fundamental sequence $z_{w}=\varphi 1 a 0[w]$ for $\varphi 1 a 0$ so that $z_{0}=1$ and $z_{w+1}=\varphi l a[w] z_{w}$, hence, (19) is an immediate consequence of (17). The proof of (20) runs similarly. Finally, (21) is straightforward as in case (b). Altogether we have concluded the verification of (18). Again, we can represent $I^{c[u]}(\varphi 1 a e)$ by a set of level $c[u]$, so that we can derive from (18) and our assumption $I^{c}(b)$ in $(14)$,

$$
(\forall u) I^{c[u]}(\varphi 1 a b) \text {. }
$$

This is literally (15) and, hence, we are done with (c). In fact, this also finishes the proof of the main lemma.

As an immediate corollary to the Main Lemma I we can state:

COROLLARY 8. The following is a theorem of $\mathrm{SRT}_{\alpha}$ :

$$
\left(\forall c, c_{0}, d\right)\left[c \preceq \alpha \wedge c=c_{0}+\omega^{1+d} \rightarrow(\forall e \prec d)(\forall b)\left(I^{c}(b) \rightarrow I^{c}(\varphi l e b)\right)\right] .
$$

A further "iteration" of the procedure of the main lemma applied to the last corollary and Corollary 6 readily entail the following corollary.

COROLLARY 9. The following is a theorem of SRT $\mathrm{S}_{\alpha}$ :

$$
\left(\forall c, c_{0}, d\right)\left[c \preceq \alpha \wedge c=c_{0}+\omega^{1+d} \rightarrow \operatorname{Prog}\left(\lambda e . I^{c}(\varphi 1 d e)\right)\right] .
$$

We also observe that we have transfinite induction available in $\mathrm{SRT}_{\alpha}$ with respect to arbitrary formulas of $\mathscr{L}_{\text {stt }}$ up to each $\beta<\varepsilon(\alpha)$.

Lemma 10. Let $\beta<\varepsilon(\alpha)$ and $A$ an arbitrary $\mathscr{L}_{\text {fix }}$ formula. Then $\operatorname{SRT}_{\alpha} \vdash \operatorname{TI}(\beta, A)$. 
Finally, the previous lemma, the previous corollary, and standard wellordering techniques for $\mathrm{SRT}_{n}$ (which in fact have already been used in the proof of Lemma 5) now yield the desired lower bound for $\mathrm{SRT}_{\alpha}$, and hence, for $\widehat{\mathrm{ID}}_{\alpha}$; this is summarized in the final theorem of this section.

THEOREM 11. Assume that $\alpha$ is an ordinal less than $\Phi_{0}$ given in the form

$$
\alpha=\omega^{1+\alpha_{n}}+\omega^{1+\alpha_{n-1}}+\cdots+\omega^{1+\alpha_{1}}+m,
$$

for ordinals $\alpha_{n} \geq \alpha_{n-1} \geq \cdots \geq \alpha_{1}$ and $m<\omega$. Then we have for all ordinals $\beta$ :

$$
\beta<\varphi 1 \alpha_{n}\left(\varphi 1 \alpha_{n-1}\left(\cdots \varphi 1 \alpha_{1}(\alpha \mid m)\right) \cdots\right) \quad \Longrightarrow \quad \widehat{\mathbb{I D}}_{\alpha} \vdash \mathrm{TI}(\beta, U) .
$$

§6. Upper bounds for $\widehat{\mathrm{ID}}_{\alpha}$. In this section we compute a sharp upper bound for $\widehat{\mathrm{ID}}_{\alpha}$ by sketching a full cut elimination theorem. We will make use of a semiformal system $\mathrm{H}_{\infty}$ and appropriate subsystems $\mathrm{H}_{\alpha}$ of $\mathrm{H}_{\infty}$. The crucial step will be Main Lemma II stated below, which is similar in spirit to the second elimination theorem of predicative proof theory. In the sequel we assume that the reader is familiar with the proof-theoretic analysis of $\widehat{\mathrm{ID}}_{n}$, i.e., the elimination of one fixed point by means of asymmetric interpretation (cf. e.g. Cantini [2], Jäger and Strahm [8], or Marzetta and Strahm [10]), or the methods presented in Feferman [5].

We start off by introducing the semiformal system $\mathrm{H}_{\infty}$. It is formulated in the language $\mathscr{L}_{\infty}$ which extends $\mathscr{L}$ by unary relation symbols $P_{\beta}^{\mathscr{A}}$ and $P_{<\beta}^{\mathscr{A}}$ for each inductive operator form $\mathscr{A}$ and each ordinal $\beta$ less than $\Phi_{0}$. The formulas of $\mathscr{L}_{\infty}$ are inductively generated as follows:

1. Every closed literal of $\mathscr{L}$ is an $\mathscr{L}_{\infty}$ formula.

2. If $t$ is a closed number term, then $P_{\beta}^{\mathscr{A}}(t), P_{<\beta}^{\mathscr{A}}(t), \sim P_{\beta}^{\mathscr{A}}(t)$ and $\sim P_{<\beta}^{\mathscr{A}}(t)$ are $\mathscr{L}_{\infty}$ formulas.

3. $\mathscr{L}_{\infty}$ formulas are closed under disjunction, conjunction, existential and universal number quantification.

Observe that $\mathscr{L}_{\infty}$ formulas do not contain free number variables. We denote $\mathscr{L}_{\infty}$ formulas that are generated according to points 1 and 2 of the preceding definition as literals of $\mathscr{L}_{\infty}$. Two such literals are called numerically equivalent, if they are syntactically identical modulo number subterms which have the same value. Further, we write pair $(t)$ if the closed term $t$ codes a pair, i.e. $t=\left\langle(t)_{0},(t)_{1}\right\rangle$ is true. Finally, for $t$ a closed term, let $|t|$ denote the unique ordinal less than $\Phi_{0}$, which is associated to $t$ with respect to the wellordering $\prec$.

The system $\mathrm{H}_{\infty}$ is formulated as a Tait-style calculus for finite sets $(\Gamma, \Delta, \ldots)$ of $\mathscr{L}_{\infty}$ formulas. If $A$ is an $\mathscr{L}_{\infty}$ formula, then $\Gamma, A$ is a shorthand for $\Gamma \cup\{A\}$, and similar for expressions of the form $\Gamma, A, B$. The system $\mathrm{H}_{\infty}$ contains the following axioms and rules of inference.

I. Axioms, group 1. For all finite sets $\Gamma$ of $\mathscr{L}_{\infty}$ formulas, all numerically equivalent $\mathscr{L}_{\infty}$ literals $A$ and $B$, and all true $\mathscr{L}$ literals $C$ :

$$
\Gamma, \neg A, B \text { and } \Gamma, C \text {. }
$$

II. Axioms, group 2. For all finite sets $\Gamma$ of $\mathscr{L}_{\infty}$ formulas, all closed terms $s$ so that pair $(s)$ is false, and all closed terms $t$ so that $\operatorname{pair}(t)$ and $\beta \leq\left|(t)_{1}\right|$ :

$$
\Gamma, \neg P_{<\beta}^{\mathscr{A}}(s) \text { and } \Gamma, \neg P_{<\beta}^{\mathscr{A}}(t) .
$$


III. Fixed point rules, group 1. For all finite sets $\Gamma$ of $\mathscr{L}_{\infty}$ formulas and all closed term $t$ so that $\operatorname{pair}(t)$ and $\left|(t)_{1}\right|=\alpha<\beta$ :

$$
\frac{\Gamma, P_{\alpha}^{\mathscr{A}}\left((t)_{0}\right)}{\Gamma, P_{<\beta}^{\mathscr{A}}(t)}, \quad \frac{\Gamma, \neg P_{\alpha}^{\mathscr{A}}\left((t)_{0}\right)}{\Gamma, \neg P_{<\beta}^{\mathscr{A}}(t)} .
$$

IV. Fixed point rules, group 2. For all finite sets $\Gamma$ of $\mathscr{L}_{\infty}$ formulas, all closed number terms $s$, and all closed number terms $t$ so that $|t|=\beta$ :

$$
\frac{\Gamma, \mathscr{A}\left(P_{\beta}^{\mathscr{A}}, P_{<\beta}^{\mathscr{A}}, s, t\right)}{\Gamma, P_{\beta}^{\mathscr{A}}(s)}, \quad \frac{\Gamma, \neg \mathscr{A}\left(P_{\beta}^{\mathscr{A}}, P_{<\beta}^{\mathscr{A}}, s, t\right)}{\Gamma, \neg P_{\beta}^{\mathscr{A}}(s)} .
$$

V. Propositional rules. For all finite sets $\Gamma$ of $\mathscr{L}_{\infty}$ formulas and all $\mathscr{L}_{\infty}$ formulas $A$ and $B$ :

$$
\frac{\Gamma, A}{\Gamma, A \vee B}, \quad \frac{\Gamma, B}{\Gamma, A \vee B}, \quad \frac{\Gamma, A \quad \Gamma, B}{\Gamma, A \wedge B} .
$$

VI. Number quantifier rules. For all finite sets $\Gamma$ of $\mathscr{L}_{\infty}$ formulas and all $\mathscr{L}_{\infty}$ formulas $A(s)$ :

$$
\frac{\Gamma, A(s)}{\Gamma,(\exists x) A(x)}, \quad \frac{\Gamma, A(t) \text { for all closed number terms } t}{\Gamma,(\forall x) A(x)}(\omega) .
$$

VII. Cut rules. For all finite sets $\Gamma$ of $\mathscr{L}_{\infty}$ formulas and all $\mathscr{L}_{\infty}$ formulas $A$ :

$$
\frac{\Gamma, A \quad \Gamma, \neg A}{\Gamma} .
$$

The formulas $A$ and $\neg A$ are the cut formulas of this cut.

Crucial for the formulation of Main Lemma II below are subsystems $\mathrm{H}_{\alpha}$ of $\mathrm{H}_{\infty}$. The system $\mathrm{H}_{\alpha}$ is formulated in the sublanguage $\mathscr{L}_{\alpha}$ of $\mathscr{L}_{\infty}$, which only allows relations $P_{\beta}^{\mathscr{A}}$ for $\beta<\alpha$ and $P_{<\beta}^{\mathscr{A}}$ for $\beta \leq \alpha$. Then $\mathrm{H}_{\alpha}$ is just the restriction of $\mathrm{H}_{\infty}$ to $\mathscr{L}_{\alpha}$.

As usual, the notion $\mathrm{H}_{\alpha} \stackrel{\beta}{\mapsto} \Gamma$ is used to express that $\Gamma$ is provable in $\mathrm{H}_{\alpha}$ by a proof of depth less than or equal to $\beta$; we write $\mathrm{H}_{\alpha} \frac{\beta}{\star} \Gamma$ if $\Gamma$ is derivable in $\mathrm{H}_{\alpha}$ by a proof of depth less than or equal to $\beta$ so that all its cut formulas are of the form $P_{\gamma}^{\mathscr{A}}(t)$ and $\neg P_{\gamma}^{\mathscr{A}}(t)$, respectively. Finally, we write $\mathrm{H}_{\alpha} \Vdash \frac{<\beta}{} \Gamma$ if there exists a $\gamma<\beta$ so that $\mathrm{H}_{\alpha} \stackrel{\nu}{-} \Gamma ; \mathrm{H}_{\alpha} \frac{<\beta}{\star} \Gamma$ is defined analogously.

One immediately realizes that the axioms and rules of $\mathrm{H}_{\alpha}$ are tailored in such a way that all cuts but the ones with respect to cut formulas of the form $P_{\beta}^{\mathscr{A}}(t)$ or $\neg P_{\beta}^{\mathscr{A}}(t)$ can be eliminated in a straightforward manner. This observation is stated in the following proposition.

Proposition 12 (Partial cut elimination for $\mathrm{H}_{\alpha}$ ). We have for all finite sets $\Gamma$ of $\mathscr{L}_{\alpha}$ formulas:

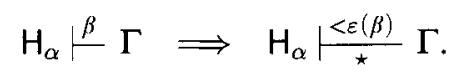

We are now in a position to formulate the main lemma of this section. Its status is similar to the one of the second elimination theorem of predicative proof theory, cf., e.g., [12]. 
LeMma 13 (Main Lemma II). Assume that $\left.\mathrm{H}_{\beta+\omega^{1+\rho}}\right|_{\star} ^{\alpha} \Gamma$ for a finite set $\Gamma$ of $\mathscr{L}_{\beta+\omega^{1+\rho}}$ formulas. Then we have for all ordinals $\xi$ less than $\omega^{1+\rho}$ :

$$
\left.\Gamma \subset \mathscr{L}_{\beta+\xi} \Longrightarrow \mathrm{H}_{\beta+\xi}\right|^{\varphi 1 \rho \alpha} \Gamma
$$

Proof. We prove the claim by main induction on $\rho$ and side induction on $\alpha$. We distinguish cases whether $\rho=0, \rho$ is a successor, or $\rho$ is a limit ordinal.

(a) $\rho=0$. Let us assume that $\Gamma$ is a finite set of $\mathscr{L}_{\beta+n}$ formulas for some natural number $n$ so that $\left.\mathrm{H}_{\beta+\omega}\right|_{\star} ^{\alpha} \Gamma$. If $\Gamma$ is an axiom of $\mathrm{H}_{\beta+\omega}$, then the claim is trivial. Furthermore, if $\Gamma$ is the conclusion of a rule different from the cut rule, the claim is immediate from the induction hypothesis. Hence, the only critical case comes up if $\Gamma$ is the conclusion of a cut rule. Then there exist a natural number $m \geq n$, $\alpha_{0}, \alpha_{1}<\alpha$ and an $\mathscr{L}_{\beta+m}$ formula $A$ of the form $P_{\delta}^{\mathscr{A}}(t)$ for some $\delta$ less than $\beta+m$ so that

$$
\mathrm{H}_{\beta+\omega} \frac{\alpha_{0}}{\star} \Gamma, A \text { and }\left.\quad \mathrm{H}_{\beta+\omega}\right|_{\star} ^{\alpha_{1}} \Gamma, \neg A \text {. }
$$

By the induction hypothesis we can conclude that

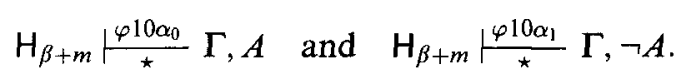

Hence, by cut we also have $\mathrm{H}_{\beta+m} \mid \frac{\gamma}{\star} \Gamma$ for $\gamma:=\max \left(\varphi 10 \alpha_{0}, \varphi 10 \alpha_{1}\right)+1$. Finally, we obtain by the standard elimination procedure of finitely many fixed points (say, by asymmetric interpretation, cf., e.g., $[2,8,10])$ that

$$
\mathrm{H}_{\beta+n} \frac{\varphi 10 \alpha}{\star} \Gamma \text {. }
$$

This proves our claim in the case $\rho=0$.

(b) $\rho=\rho_{0}+1$. Let $\xi<\omega^{1+\rho_{0}+1}$ and $\Gamma$ be a finite set of $\mathscr{L}_{\beta+\xi}$ formulas so that

$$
\mathrm{H}_{\beta+\omega^{1+p_{0}+1}} \frac{\alpha}{\star} \Gamma \text {. }
$$

Observe that $\xi=\omega^{1+\rho_{0}} \cdot n+\eta$ for some natural number $n$ and some $\eta<\omega^{1+\rho_{0}}$. Again the only crucial case occurs if $\Gamma$ is the conclusion of a cut. Then there exist a natural number $m>n, \alpha_{0}, \alpha_{1}<\alpha$ and an $\mathscr{L}_{\beta+\omega^{1+p_{0} \cdot m}}$ formula $A$ of the form $P_{\delta}^{\mathscr{A}}(t)$ for some $\delta$ less than $\beta+\omega^{1+\rho_{0}} \cdot m$ so that

$$
\mathrm{H}_{\beta+\omega^{1+\rho_{0}+1}} \frac{\alpha_{0}}{\star} \Gamma, A \text { and } \mathrm{H}_{\beta+\omega^{1+\rho_{0}+1}} \frac{\alpha_{1}}{\star} \Gamma, \neg A \text {. }
$$

By applying the side induction hypothesis to (5) we derive

$$
\mathbf{H}_{\beta+\omega^{1+\rho_{0} \cdot m}} \frac{\varphi 1 \rho \alpha_{0}}{\star} \Gamma, A \quad \text { and } \quad \mathrm{H}_{\beta+\omega^{1+\rho_{0} \cdot m}} \frac{\varphi 1 \rho \alpha_{1}}{\star} \Gamma, \neg A \text {, }
$$

and hence, $\mathrm{H}_{\beta+\omega^{1+\rho_{0} \cdot m}} \frac{\gamma}{\star} \Gamma$ for $\gamma:=\max \left(\varphi 1 \rho \alpha_{0}, \varphi \mathrm{l} \rho \alpha_{1}\right)+1$. If we inductively define a sequence of ordinals $\gamma_{i}$ by $\gamma_{0}:=\gamma$ and $\gamma_{i+1}:=\varphi 1 \rho_{0} \gamma_{i}$, then one readily 
obtains by $m-n$ times applying the main induction hypothesis:

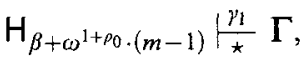

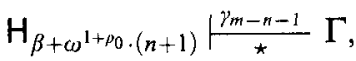

$$
\begin{aligned}
& \mathrm{H}_{\beta+\omega^{1+\rho_{0} \cdot n+\eta}} \frac{\gamma_{m-n}}{\star} \Gamma \text {. }
\end{aligned}
$$

Here we have successively replaced $\beta$ by

$$
\beta+\omega^{1+\rho_{0}} \cdot(m-1), \quad \ldots, \quad \beta+\omega^{1+\rho_{0}} \cdot(n+1), \quad \beta+\omega^{1+\rho_{0}} \cdot n
$$

in the main induction hypothesis. Since $\gamma_{m-n}<\varphi 1 \rho \alpha$, we have indeed established

$$
\mathrm{H}_{\beta+\xi} \frac{\varphi 1 \rho \alpha}{\star} \Gamma
$$

as desired. This finishes the treatment of the successor case.

(c) $\rho$ is limit. Assume that $\xi<\omega^{1+\rho}$ and $\Gamma$ is a finite set of $\mathscr{L}_{\beta+\xi}$ formulas so that

$$
\left.\mathrm{H}_{\beta+\omega^{\rho}}\right|_{\star} ^{\alpha} \Gamma \text {. }
$$

Again assume that $\Gamma$ is the conclusion of cut. Then there exists $\rho_{0}<\rho$ with $\xi \leq \omega^{1+\rho_{0}}, \alpha_{0}, \alpha_{1}<\alpha$ and an $\mathscr{L}_{\beta+\omega^{1+\rho_{0}}}$ formula $A$ of the form $P_{\delta}^{\mathscr{A}}(t)$ for some $\delta$ less than $\beta+\omega^{1+\rho_{0}}$ so that

$$
\mathrm{H}_{\beta+\omega^{p}} \frac{\alpha_{0}}{\star} \Gamma, A \quad \text { and } \quad \mathrm{H}_{\beta+\omega^{p}} \vdash_{\star}^{\alpha_{1}} \Gamma, \neg A \text {. }
$$

The side induction hypothesis applied to (9) and (10) produces

$$
\mathrm{H}_{\beta+\omega^{\rho}} \frac{\varphi \mathrm{l} \rho \alpha_{0}}{\star} \Gamma, A \text { and } \mathrm{H}_{\beta+\omega^{\rho}} \mid \frac{\varphi 1 \rho \alpha_{1}}{\star} \Gamma, \neg A \text {, }
$$

which by a cut yields $\left.H_{\beta+\omega^{\rho}}\right|_{\star} ^{\gamma} \Gamma$ for $\gamma:=\max \left(\varphi 1 \rho \alpha_{0}, \varphi 1 \rho \alpha_{1}\right)+1$. From this we conclude by the main induction hypothesis

$$
\mathbf{H}_{\beta+\xi} \frac{\varphi 1 p_{0} \gamma}{\star} \Gamma \text {. }
$$

Since $\varphi 1 \rho_{0} \gamma<\varphi 1 \rho \alpha$, this is our claim. This finishes the proof of (c) and also the verification of the main lemma.

As an immediate corollary we obtain the following result.

COROLLARY 14. Assume that $\left.\mathrm{H}_{\beta+\omega^{1+\rho}}\right|_{\star} ^{\alpha} \Gamma$ for a finite set $\Gamma$ of $\mathscr{L}_{\beta}$ formulas. Then we have $\mathrm{H}_{\beta} \frac{\varphi l \rho \alpha}{\star} \Gamma$.

Our upper bound computation for $\widehat{\mathrm{ID}}_{\alpha}$ is nearly complete. What is still missing is the embedding of $\widehat{\mathrm{ID}}_{\alpha}$ into $\mathrm{H}_{\alpha}$; this is obtained in an obvious manner by interpreting the predicate $P^{\mathscr{A}}$ by $P_{<\alpha}^{\mathscr{A}}$, thus establishing a translation $(\cdot)^{\alpha}$ of $\mathscr{L}_{\text {fix }}$ into $\mathscr{L}_{\alpha}$. The embedding theorem can now be given straightforwardly; in the formulation below we have directly combined it with Proposition 12. Since the proof is standard, we will omit it.

Proposition 15 (Embedding of $\widehat{\mathrm{ID}}_{\alpha}$ into $\mathrm{H}_{\alpha}$ ). We have for all $\mathscr{L}_{\text {fix }}$ sentences $A$ :

$$
\widehat{\mathrm{ID}}_{\alpha} \vdash A \Longrightarrow \mathrm{H}_{\alpha} \stackrel{<\varepsilon(\alpha)}{\star} A^{\alpha} \text {. }
$$


From the previous corollary, the embedding theorem, and standard elimination techniques for finitely many fixed points (which actually have already been used in the proof of Main Lemma II) we are now in a position to state the full cut elimination theorem for $\widehat{\mathrm{ID}}_{\alpha}$.

THEOREM 16. Assume that $\alpha$ is an ordinal less than $\Phi_{0}$ given in the form

$$
\alpha=\omega^{1+\alpha_{n}}+\omega^{1+\alpha_{n-1}}+\cdots+\omega^{1+\alpha_{1}}+m,
$$

for ordinals $\alpha_{n} \geq \alpha_{n-1} \geq \cdots \geq \alpha_{1}$ and $m<\omega$. If $\delta$ denotes the ordinal

$$
\left.\varphi 1 \alpha_{n}\left(\varphi 1 \alpha_{n-1}\left(\ldots \varphi 1 \alpha_{1}(\alpha \mid m)\right) \ldots\right)\right),
$$

then we have for all $\mathscr{L}$ sentences $A$ :

$$
\widehat{\mathrm{ID}}_{\alpha} \vdash A \Longrightarrow \mathrm{H}_{0} \vdash \frac{<\delta}{\star} A \text {. }
$$

Of course, this theorem gives the desired upper bound for the proof-theoretic ordinal of $\widehat{\mathrm{ID}}_{\alpha}$, so that we have now established a complete proof of the Main Theorem of this article as it has been stated in Section 3.

§7. Final remarks. If $\alpha$ is an ordinal less $\Phi_{0}$, then we write $\widehat{I D}_{<\alpha}$ for the union of the theories $\widehat{\mathrm{ID}}_{\beta}$ for $\beta<\alpha$. Then it follows immediately that the proof-theoretic ordinal of $\widehat{\mathrm{ID}}_{<\alpha}$ is the supremum of the proof-theoretic ordinals of the theories $\widehat{\mathrm{ID}}_{\beta}$ with $\beta<\alpha$. For example, for the ordinals $\omega, \omega^{\omega}$ and $\varepsilon_{0}$ we obtain from our Main Theorem:

COROLLARY 17. We have the following proof-theoretic ordinals:

$$
\begin{aligned}
& \left|\widehat{I D}_{<\omega}\right|=\varphi 100=\Gamma_{0}, \quad\left|\widehat{I D}_{\omega}\right|=\varphi 10 \varepsilon_{0}=\Gamma_{\varepsilon_{0}}, \\
& \left|\widehat{I D}_{<\omega^{\omega}}\right|=\varphi 1 \omega 0, \quad\left|\widehat{\mathbb{D}}_{\omega^{\omega}}\right|=\varphi 1 \omega \varepsilon_{0} \text {, } \\
& \left|\widehat{\mathrm{ID}}_{<\varepsilon_{0}}\right|=\varphi 1 \varepsilon_{0} 0, \quad\left|\widehat{\mathrm{ID}}_{\varepsilon_{0}}\right|=\varphi 1 \varepsilon_{0} \varepsilon_{1} .
\end{aligned}
$$

In Jäger and Strahm [7] some proof-theoretic equivalences between transfinitely iterated fixed point theories and subsystems of second order arithmetic are established. It is shown in particular that

$$
\widehat{\mathrm{ID}}_{\omega} \equiv \mathrm{ATR}, \quad \widehat{\mathrm{ID}}_{<\omega^{\omega}} \equiv \mathrm{ATR}_{0}+\left(\Sigma_{1}^{1}-\mathrm{DC}\right), \quad \widehat{\mathrm{ID}}_{<\varepsilon_{0}} \equiv \mathrm{ATR}+\left(\Sigma_{1}^{1}-\mathrm{DC}\right) .
$$

A further result about the theories $\widehat{I D}_{\alpha}$, which will be published elsewhere, refers to the autonomous closure of fixed point theories.

There exist also close connections between iterated fixed point theories and Martin-Löf type theories with universes. The finite case is completely treated in Feferman [5]. Stronger principles concerning universes make use of so-called superuniverses as discussed, e.g., in Palmgren [11]. Several of these have been proof-theoretically treated by Rathjen [13]. Furthermore, the theories $\widehat{I D}_{\alpha}$ are also closely related to Frege structures (cf. Kahle [9]) and certain systems of explicit mathematics with universes (cf. Strahm [16]).

From a more general point of view, one can point out that the theories $\widehat{\mathrm{ID}}_{\alpha}$ for $\alpha \geq \omega$ provide examples of metapredicative theories, i.e., theories, which have proof-theoretic ordinal beyond $\Gamma_{0}$ but can still be treated by methods of predicative proof theory. In a sense, the theories $\widehat{\operatorname{ID}}_{\alpha}(\alpha \geq \omega)$ can be used to scale the initial 
part of metapredicative theories in the same sense as the theories $\operatorname{ID}_{\alpha}(\alpha \geq 1)$ could be used to scale the initial part of impredicative theories.

\section{REFERENCES}

[1] W. Buchholz, S. Feferman, W. Pohlers, and W. SieG, Iterated inductive definitions and subsystems of analysis: Recent proof-theoretical studies, Lecture Notes in Mathematics, vol. 897, Springer-Verlag, Berlin, 1981.

[2] A. CANTINI, A note on a predicatively reducible theory of iterated elementary induction, Bollettino Unione Mathematica Italiana 4-B, vol. 6 (1985), pp. $413-430$.

[3] - Notes on formal theories of truth, Zeitschrift für mathematische Logik und Grundlagen der Mathematik, vol. 35 (1989), pp. 97-130.

[4] — Logical frameworks for truth and abstraction, North-Holland, Amsterdam, 1996.

[5] S. FefERman, Iterated inductive fixed-point theories: application to Hancock's conjecture, The Patras symposion (G. Metakides, editor), North-Holland, Amsterdam, 1982, pp. 171-196.

[6] - Reflecting on incompleteness, this JourNAL, vol. 56 (1991), no. 1, pp. 1-49.

[7] G. JÄGER and T. STrahm, Fixed point theories and dependent choice, Archive for Mathematical Logic, to appear.

[8] - Some theories with positive induction of ordinal strength $\varphi \omega 0$, this JOURNAL, vol. 61 (1996), no. 3, pp. $818-842$.

[9] R. Kahle, Applicative theories and Frege structures, Ph.D. thesis, Institut für Informatik und angewandte Mathematik, Universität Bern, 1997.

[10] M. MARZETTA and T. STRAHM, The $\mu$ quantification operator in explicit mathematics with universes and iterated fixed point theories with ordinals, Archive for Mathematical Logic, vol. 37 (1998), pp. $391-413$.

[11] E. Palmaren, On universes in type theory, Twenty-five years of type theory (G. Sambin and J. Smith, editors), Oxford University Press, to appear.

[12] W. PoHLERs, Proof theory: An introduction, Lecture Notes in Mathematics, vol. 1407, SpringerVerlag, Berlin, 1988.

[13] M. RATHJEN, The strength of Martin-Löf type theory with a superuniverse, Part I, preprint, 1997.

[14] K. SCHËTTE, Kennzeichnung von Ordnungszahlen durch rekursiv erklärte Funktionen, Mathematische Annalen, vol. 127 (1954), pp. 15-32.

[15] — Proof theory, Springer-Verlag, Berlin, 1977.

[16] T. STRAHM, First steps into metapredicativity in explicit mathematics, to appear.

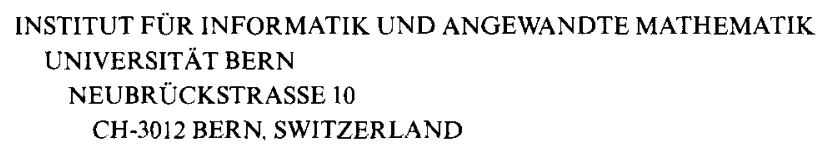

E-mail: jaeger@iam.unibe.ch

UNIVERSITÄT TÜBINGEN. WSI

SAND 13

D-72076 TÜBINGEN, GERMANY

E-mail: kahle@informatik.uni-tuebingen.de

DEPARTMENT OF MATHEMATICS

UPPSALA UNIVERSITY

P.O. BOX 480

S-751 06 UPPSALA, SWEDEN

E-mail: setzer@math.uu.se

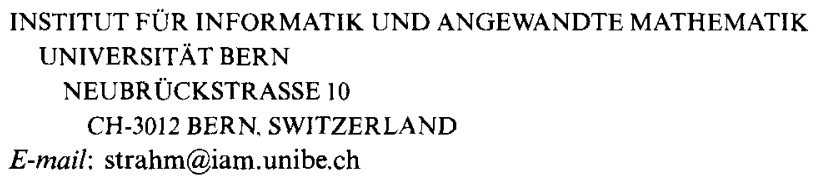

